\title{
Nanostructure of Cationic Polymer Brush at the Air/Water Interface
}

\author{
Hideki Matsuoka*1, Shin'ichi Fujita ${ }^{1}$, Arjun Ghosh ${ }^{1}$, Shunichi Nakayama ${ }^{1}$, Yuta Yamakawa ${ }^{1}$, Shin-ichi Yusa ${ }^{2}$ \\ and Yoshiyuki Saruwatari ${ }^{3}$ \\ ${ }^{1}$ Department of Polymer Chemistry, Kyoto University, Katsura, Kyoto 615-8510, Japan \\ ${ }^{2}$ Graduate School of Engineering, University of Hyogo, 2167 Shosha, Himeji, Hyogo 671-2280, Japan \\ ${ }^{3}$ Osaka Organic Chemical Industry, 1-7-2 Hon-cho, Nihonbashi, Chuo-ku, Tokyo 103-0023 \\ *To whom correspondence should be addressed. matsuoka@star.polym.kyoto-u.ac.jp
}

\begin{abstract}
Cationic amphiphilic diblock copolymers were synthesized by RAFT polymerization and the nanostructure of their monolayers was investigated by $\pi$ - $A$ isotherm and X-ray reflectivity. Carpet layer (dense hydrophilic block layer) formation under the hydrophobic layer was confirmed and a "brush" layer was found beneath the carpet layer. However, the thickness of brush layer was much thinner than that of the fullystretched chain length. The critical salt concentration was found to be $0.01 \mathrm{M} \mathrm{NaCl}$, which is much lower than that of the previous strongly anionic brush. These differences were probably caused by the low effective charge on the brush chains due to the hydrophobic nature of the quarternized ammonium cation.
\end{abstract}

\section{Introduction}

Polyelectrolyte brushes have been attracting much attention as a novel surface modification technique for improvement of friction, biocompatibility etc.[1-7] We have been investigating the nanostructure and the transition of the polyelectrolyte brush in the ionic amphiphilic diblock copolymer monolayer at the air/water interface by utilizing surface pressure - area $(\pi$ A) isotherm and X-ray and neutron reflectivity techniques.[8-13] The primary advantage of monolayer systems for brush study is easy control/change of brush density (number of brush chains in unit area) and precise control of molecular weight and the distribution of brush chains. So far, we have been studying the anionic brush systems, i.e., poly(methacrylic acid) (PMA)[8-11], poly(styrenesulfonate) (PSS)[12], and poly(acrylic acid) (PAA)[13]. The absolute value of the critical brush density (the brush density where transition from carpetonly structure to carpet+brush structure occurs) and its ionic chain length dependence were found to be different for these three anionic brush systems. In addition, there was a critical salt concentration for strongly ionic PSS brush where the brush chains start to shrink [12]. The weak acid brushes such as PMA and PAA brushes showed different added salt concentration dependence; the brush chains first expanded and then shrunk with increasing added salt concentration.[13] From these observations, we concluded that the brush formation mechanism is different for PMA, PSS, and PAA brushes.[13] In addition, there was a different critical salt concentration for PSS brush for different kinds of added salt, e.g., $\mathrm{NaCl}, \mathrm{KCl}$, and $\mathrm{LiCl}$, which follows the Hofmeister series, which reminds us of the special situation of water structure in the polyelectrolyte brush layer which has a high ion concentration.[14]

In this study, we investigated cationic brush systems using the cationic amphiphilic diblock copolymer ( $\mathrm{P}(\mathrm{QBm})-b-\mathrm{P}(\mathrm{nBA})$, and $\mathrm{P}(\mathrm{QBm})-b-\mathrm{P}(\mathrm{EHA})$ monolayer on the water surface. A systematic study was conducted using $\pi-A$ isotherm and $\mathrm{X}$-ray reflectivity (XR).

\section{Materials and Methods}

\subsection{Materials}

\subsubsection{Monomers and chemicals}

The cationic monomer, (4-vinylbenzyl)trimethylammonium chloride $(\mathrm{QBm})$, and hydrophobic monomer, 2-ethylhexyl acrylate (EHA), were kind gifts from Osaka Organic Chemical Industry. Other hydrophobic monomer, $n$-butyl acrylate (nBA) was a product of Tokyo Kasei. $n \mathrm{BA}$ and EHA were distilled under a low pressure with $\mathrm{LiH}$ before use. The initiators, 4,4'-azobis(isobutyronitrile) (AIBN) and 4,4'azobis(cyanopentanoic acid) (ACVA) (98\%) were purchased from Wako (Osaka, Japan). Other chemicals were also reagent grade Wako products and used as received. Deuterated solvents for NMR measurements were EURISO-TOP (98\%). Water used was ultrapure 
water obtained using the Milli-Q system. Dialysis tube was a product of Orange Scientific (MWCO:3500).

\subsubsection{Chain transfer agent (CTA)}

The chain transfer agent (CTA) for RAFT polymerization was 4-cyanopentanoic acid dithiobenzoate, which was synthesized as reported.[15,16]

\subsection{Polymer synthesis}

The block copolymers were synthesized by reversible addition fragmentation chain transfer (RAFT) process. (Scheme 1) First, QBm homopolymers were synthesized. QBm, CTA, and ACVA were dissolved in a water/DMF mixture in a Schlenk tube and degassed by three freezepump-thaw cycles. After the polymerization reaction in a $70^{\circ} \mathrm{C}$ oil bath for 2 hours, the polymer obtained was purified by dialysis and lyophilized.

By using QBm homopolymer as a macro-CTA, block copolymers were synthesized. PQBm, AIBN, and nBA or EHA were dissolved in methanol in Schlenk tube and treated with three cycles freeze-pump-thaw. After kept at $70^{\circ} \mathrm{C}$ for 24 hours for polymerization, the solution was poured in hexane to precipitate block copolymers. The polymers obtained were dried under vacuum. Further details on the polymer synthesis can be found in Supporting Information (SI).

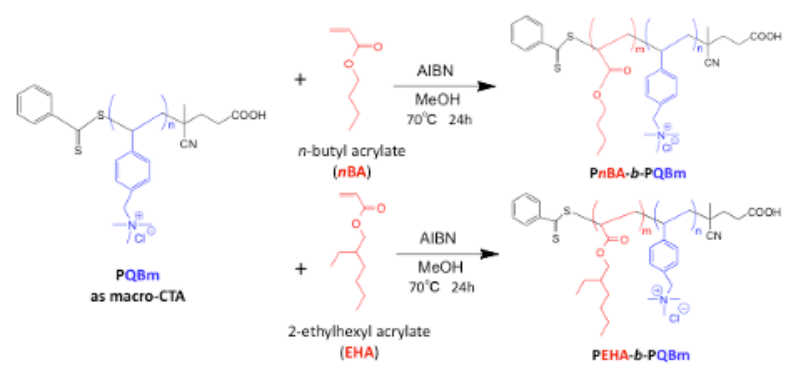

Scheme 1. Block copolymer synthesis.

\subsection{Methods}

\subsubsection{NMR}

${ }^{1} \mathrm{H}$ NMR was measured by a JEOL 400WS (JEOL, Japan). For QBm homopolymer, $\mathrm{D}_{2} \mathrm{O}$ was used as a solvent and for block copolymers, $\mathrm{CDCl}_{3}$ and $\mathrm{CD}_{3} \mathrm{OD}$ mixture (3/1, $\mathrm{v} / \mathrm{v}$ ) was used.

\subsubsection{GPC}

GPC measurements were performed with a JASCO (Tokyo, Japan) system with a refractive index detector (RI-2021 Plus), UV detector (UV-2075 Plus), Shodex column (SB-804 HQ), acetic acid (0.5M) and sodium sulfate $(0.3 \mathrm{M})$ buffer solution $(\mathrm{pH} 3)$ as eluent and calibration using a poly(vinylpyrridine) standard.

\subsection{3 $\pi$-A isotherm}

$\pi-\mathrm{A}$ isotherm was measured by a specially designed Langmuir-Brodgett (LB) trough made by USI System (Fukuoka, Japan), equipped with an XR instrument.

\subsubsection{XR}

The XR instrument for the water surface study was Rigaku RINT-TTR-MA reflectometer (Rigaku, Tokyo, Japan) equipped with a specially designed LB trough. Details of the instrument[17], method, and data analysis $[9,11]$ are fully described elsewhere.

\subsubsection{Brewster Angle Microscopy}

Brewster angle microcopy (BAM) observation was carried out with a Multiskop system of Optrel (Sinzing, Germany)[18] with a specially designed LangmuirBrodgett (LB) trough made by USI System.

\section{Results and Discussion}

\subsection{Polymer Characterization}

${ }^{1} \mathrm{H}$ NMR spectra for QBm homopolymer, $\mathrm{P}(\mathrm{QBm})-b$ $\mathrm{P}(\mathrm{nBA})$, and $\mathrm{P}(\mathrm{QBm})-b$-P(EHA) block copolymers are shown in figure $\mathrm{S} 1$ in SI. Each peak can be assigned as shown in the figure. From the molecular weight of QBm homopolymer by GPC and peak area ratio calculated from figure $\mathrm{S} 1$, the degree of polymerization of hydrophilic (cationic) and hydrophobic blocks $(m: n)$ were estimated as shown in table 1 .

Table 1. Characteristics of polymers synthesized.

\begin{tabular}{|c|c|c|c|c|}
\hline Polymer & $\mathrm{DP}^{b)}$ & & $M \mathrm{w} / M \mathrm{n}^{b)}$ & \\
\hline PQBm & $\begin{array}{r}52 \\
55 \\
147\end{array}$ & & $\begin{array}{l}1.21 \\
1.19 \\
1.21\end{array}$ & \\
\hline Polymer & & $m^{a)}$ & $: n^{b)}$ & $M \mathrm{n}^{c)}$ \\
\hline $\mathrm{P}(\mathrm{nBA})-b-\mathrm{p}(\mathrm{QBm})$ & & $\begin{array}{l}139 \\
245\end{array}$ & $\begin{array}{c}: 55 \\
: 147\end{array}$ & $\begin{array}{l}30100 \\
64400\end{array}$ \\
\hline $\mathrm{P}(\mathrm{EHA})-b-\mathrm{P}(\mathrm{QBm})$ & & $\begin{array}{l}141 \\
230\end{array}$ & $\begin{array}{l}: 52 \\
: \quad 52\end{array}$ & $\begin{array}{l}30100 \\
54000\end{array}$ \\
\hline
\end{tabular}

a) determined by ${ }^{1} \mathrm{H}$ NMR (peak $a$ and $d$ )

b) determined by GPC, c) calculated by $m$ and $n$ values

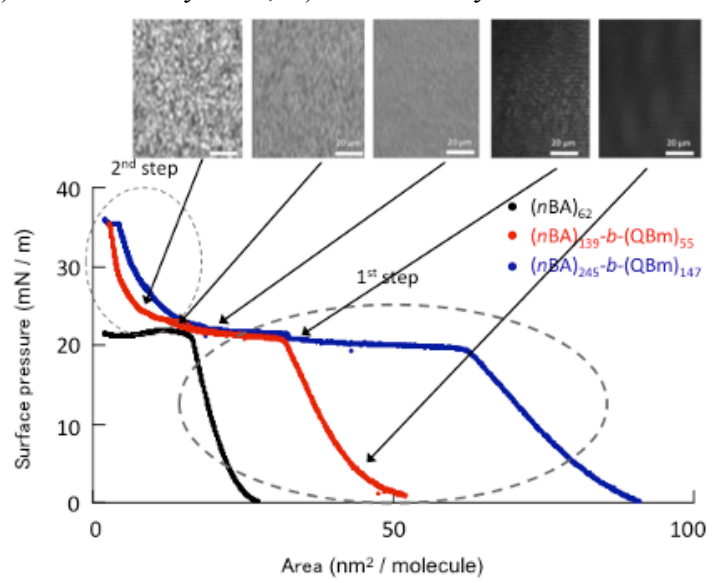

Fig.1 $\pi-A$ isotherms and BAM images for $\mathrm{P}(\mathrm{nBA})-b$ $\mathrm{P}(\mathrm{QBm})$ block copolymer monolayers on the water surface. Black line is isothem for $\mathrm{P}(\mathrm{nBA})$ homopolymer. Data for other polymer is in SI. 


\section{$3.2 \pi-A$ isotherm and the salt effect on surface pressure}

Chloroform/methanol (9/1, v/v) solution of block copolymer was spread on the water surface to form a monolayer. Figure 1 shows the $\pi-A$ isotherms for $\mathrm{P}(\mathrm{nBA})-b-\mathrm{P}(\mathrm{QBm})$ monolayers together with that for hydrophobic homopolymer and figure $\mathrm{S} 2$ shows those for the EHA polymer. In both cases, a two-step increase of $\pi$ with a large plateau region was observed, which is different from previous cases in which poly(hydrogenated isoprene) was used as a hydrophobic block. This might be special characteristics of poly(acrylate) and poly(methacrylate) analogue chains as hydrophobic block, but this is our of the scope of this study. The isotherm for the hydrophobic homopolymer shows only the first increase of $\pi$ with a plateau region. Thus, this part was from the hydrophobic block and the second increase was due to polyelectrolyte brush formation. By BAM observation, some domain structure was observed in both cases: This behaviour may be typical for acrylate polymers, but this is out of the scope of this study since our interest is the polymer brush. Figure S3 shows the added salt $(\mathrm{NaCl})$ concentration dependence of $\pi-A$ isotherm. In both cases, the isotherm shifted towards smaller regions with increasing salt concentration. This behaviour is similar to that for a strong acid PSS brush, which can be explained by the shielding effect for electrostatic repulsion between ionic brush chains by salt ions, but different from that for weak acid brushes (PMA and PAA brushes).

Figure 2 shows the added salt concentration dependence of surface pressure $\pi$ for area $A$. $\pi$ was almost constant (even slightly increased) to a certain salt concentration and then decreased rapidly. This is the same as observed for the PSS brush, which is strongly anionic. Hence, the PQBm brush behaves as a strongly cationic brush, in this sense. The bending point of $\pi$ with salt is about $0.01 \mathrm{M}$ of $\mathrm{NaCl}$, which should be the "critical salt concentration (csc)" for PQBm brush. This value is quite interesting since this is very small compared with csc for the PSS brush, 0.2M.[12] We will discuss this point after watching the PQBm brush nanostructure and its transition directly using the XR technique.

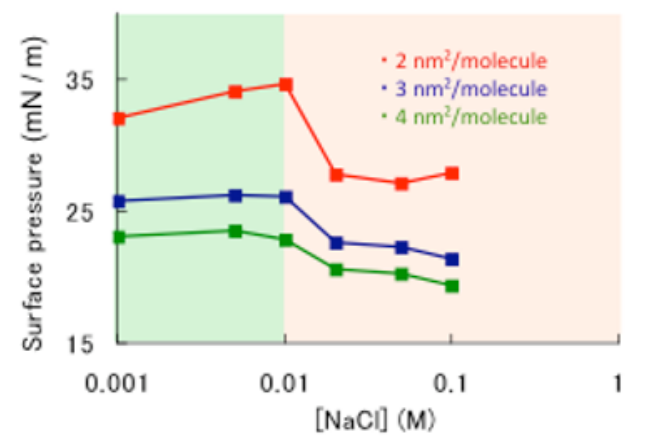

Fig.2 Added salt concentration dependence of surface pressure for (EHA) $)_{141}-b-(\mathrm{QBm})_{52}$ in an area of 2, 3 and 4 $\mathrm{nm}^{2} /$ molecule. Data for other polymers are shown in SI.

\subsection{X-ray reflectivity}

Figure 3 shows the XR profiles for three cationic block copolymer monolayers on the water surface and density profiles of the monolayer normal to the surface evaluated by model fitting of XR profiles. XR profiles were well fitted by the 3-box model consisting of hydrophobic layer on the water surface, dense hydrophilic carpet layer just beneath the water surface, and cationic brush layer under the carpet layer. The carpet layer formation (to avoid direct contact between hydrophobic layer and water subphase) and brush layer formation show the same behavior as for other anionic amphiphilic block copolymers, which confirms that this is a universal phenomenon for ionic brush in monolayer systems.
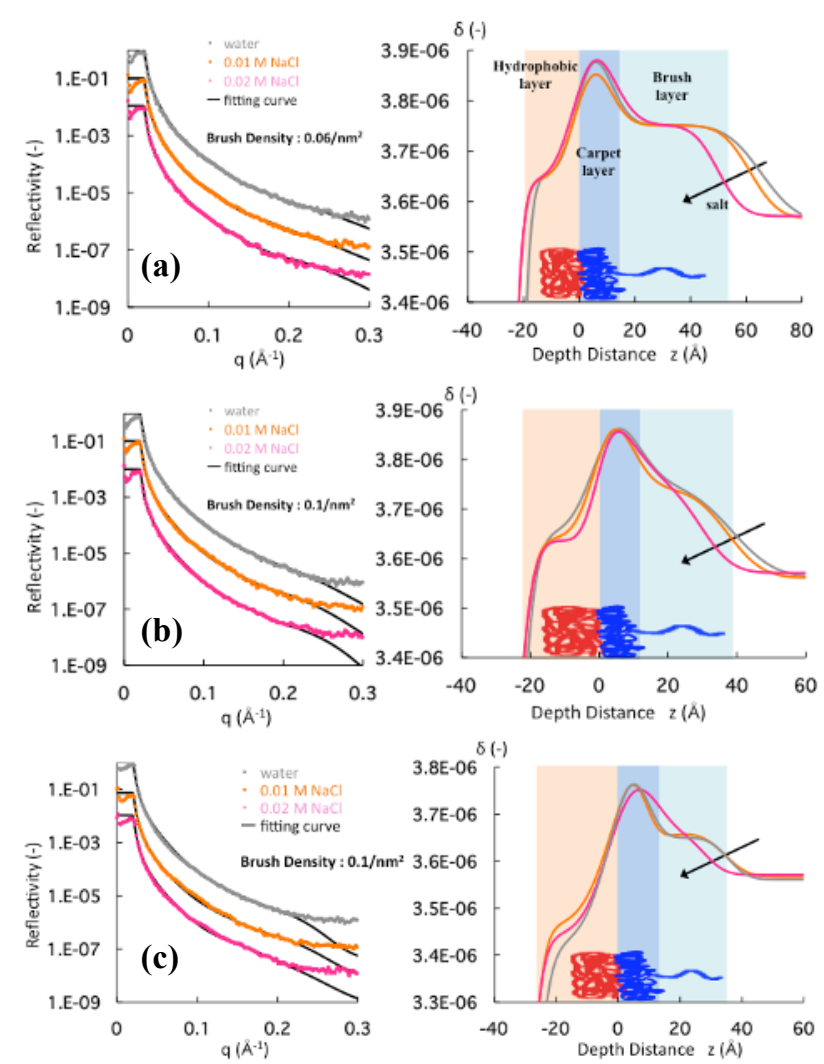

Fig.3 XR profiles (left) and density profiles (right) for (a) of $(n \mathrm{BA})_{245}-b-(\mathrm{QBm})_{147}$, (b) $(n \mathrm{BA})_{139}-b-(\mathrm{QBm})_{55}$, and of $(\mathrm{EHA})_{141}-b-(\mathrm{QBm})_{52}$ monolayers on the water surface with different added salt concentrations. $q$ is the scattering vector $(=4 \pi \sin \theta / \lambda, 2 \theta$ :scattering angle, $\lambda$ :wavelength of $\mathrm{X}$-ray $(1.5406 \AA) . z$ is the depth from water surface and $\delta$ is a parameter proportional to electron density $(n=1-\delta-i \beta, n$ : refractive index).

In the no salt condition, brush layer is formed and its thickness is almost the same as that in the $0.01 \mathrm{M} \mathrm{NaCl}$ condition. However, it is obvious that brush chains are shrunken in a $0.02 \mathrm{M} \mathrm{NaCl}$ condition. This behaviour is consistent with the salt concentration dependence of $\pi$ discussed above. Hence, the csc for PQBm brush can be said to be between $0.01 \mathrm{M}$ and $0.02 \mathrm{M} \mathrm{NaCl}$.

The csc for PSS brush was $0.2 \mathrm{M}$ for $\mathrm{NaCl}$.[12] The csc is the salt concentration where the ion (salt) concentration in the bulk subphase becomes larger than the "effective" 
ion concentration in brush layer. The analytical ion concentration in the brush layer can be calculated from the brush density and brush layer thickness, and was estimated to be about 2.2 M for the PSS brush. Since the "effective" ion concentration is $0.2 \mathrm{M}$, this means that $92 \%$ of counterions for PSS brush are condensed by the counterion condensation effect[19], which results in an effective ion concentration of $0.2 \mathrm{M}$. This anomalously large counterion condensation was attributed to the very high ion concentration in the brush layer (2.2M).[12] In the present $\mathrm{PQBm}$ brush case, the brush density is about 0.1 chain $/ \mathrm{nm}^{2}$ and the analytical ion concentration in the brush was estimated to be $1.6 \mathrm{M}$. Since the "effective" ion concentration in the brush should be $0.01 \mathrm{M}$, the degree of counterion condensation in $\mathrm{PQBm}$ brush was calculated to be more than $99 \%$, which is abnormally high.

Another important aspect is the degree of brush chain stretching. For the PSS brush, the brush layer thickness was about $78 \%$ of the fully stretched PSS chain, which can be said to be well stretched. For the PQBm brush, this brush stretch parameter was evaluated to be only $25 \%$. Hence, the PQBm brush is not stretched out, and it has a "mushroom" structure rather than a "brush" structure. The reason for this is not clear at this stage, but one should take into account the special nature of quarternized ammonium cation. Inorganic ions such as metal ions can be classified to be I and II, which is a structure maker and breaker ions, respectively. The quarternized ammonium ions are categorized into class III due to their large size and high hydrophobicity.[20,21] This special nature should contribute to the observed phenomenon, which needs to be studied further.

\section{Conclusions}

Nanostructure and the transition of the cationic polyelectrolyte brush in the water surface polymer monolayer was investigated by the $\pi-A$ isotherm and XR techniques as a function of brush density and added salt concentration. The hydrophilic cationic block forms a carpet/brush double-layer structure like other anionic brush systems. The response to salt addition was similar to that of the strongly anionic PSS brush, but the csc was lower by order of magnitude, probably due to the very high counterion condensation and showed a mushroom structure rather than a stretched out brush structure. The hydrophobic nature of the quarternized cation is thought to be what makes the structure different from that of the strongly anionic brush systems.

\section{Acknowledgement}

This work was supported by a Grant-in-Aid for Scientific Research on Innovative Areas "Molecular Soft-Interface Science" from the Ministry of Education, Culture, Sports, Science and Technology of Japan. The authors would like to express sincere thanks to Professor Ken-ici Iimura (Utsunomiya University, Japan) for his kind help in the BAM observation.

\section{References}

1 R.C. Advincula, W.J. Brittain, K.C. Caster, J.Rühe (Eds.) "Polymer Brushes" Wiley, (2004).

2 S.T.Milner, Science, 251, 905 (1991).

3 A.Halperin, M.Tirrell, T.P.Lodge, Adv. Polym. Sci., 100, 31 (1992).

4 E.B.Zhulina, O.V.Borisov, V.A.Priamitsyn, J. Colloid Interface Sci., 137, 495 (1990)

5 Y.Tran, P.Auroy, L.T. Lee, Macromolecules, 32, 8952 (1999).

6 H.Ahrens, S.Förster, C.A.Helm, Phys. Rev. Lett., 81, 4172 (1998).

7 U.Raviv, S.Giasson, N.Kampf, J.F.Gohy, R.Jerome, J.Klein, Nature (London), 425, 163 (2003).

8 E.Mouri, K.Matsumoto, H.Matsuoka J.Polym.Sci.,B, 41, 1921 (2003).

9 E.Mouri, Y.Furuya, K.Matsumoto, H.Matsuoka, Langmuir, 20, 8062 (2004).

10 (a)H.Matsuoka, Y.Furuya, P.Kaewsaiha, K.Matsumoto, Langmuir, 21, 6845 (2005), (b) H.Matsuoka, Y.Furuya, P.Kaewsaiha, K.Matsumoto, Macromolecules, 40, 766 (2007).

11 (a) E.Mouri, P.Kaewsaiha, K.Matsumoto, H.Matsuoka, N.Torikai, Langmuir, 20, 10604 (2004), (b) E.Mouri, K.Matsumoto, H.Matsuoka, N.Torikai, Langmuir, 21, 1840 (2005).

12 (a) P.Kaewsaiha, K.Matsumoto, H.Matsuoka, Langmuir, 20, 6754 (2004), (b) P.Kaewsaiha, K.Matsumoto, H.Matsuoka, Langmuir, 23, 20 (2007). (c) P.Kaewsaiha, K.Matsumoto, H.Matsuoka, Langmuir, 23, 7065 (2007).

13 H.Matsuoka, Y.Suetomi, P.Kaewsaiha, K.Matsumoto, Langmuir, 25, 13752 (2009).

14 H.Matsuoka, S.Nakayama, T.Yamada, Chem.Lett, 41, 1060 (2012).

15 (a) Y.Mitsukami, M.S.Donovan, A.B.Lowe, C.L.McCormick, Macromolecules, 34, 2248 (2001). (b) S.Oae, T.Yagihara, T.Okabe, Tetrahedron, 28, 3203 (1972).

16 A.Ghosh, S.-i. Yusa, H.Matsuoka, Y.Saruwatari, Langmuir, 27, 9237 (2011).

17 H.Matsuoka, E.Mouri, K.Matsumoto, Rigaku Journal, 18, 54 (2001).

18 H.Harke, R.Teppner, O.Schulz, H.Orendi, R.Motschmann, Rev.Sci.Instrum., 68, 3130 (1997).

19 G.S.Manning, J.Chem.Phys., 51, 924 (1969).

20 H.Ohtaki, Ion no Suiwa (Ion hydration); Kyoritsu: Tokyo (1990); in Japanese

21 R.A.Robinson, R.H.Stokes, Electrolyte Solutions, Butterworth, 1959. 